\title{
Correction to: The prevalence and risk factors of peripheral neuropathy among patients with type 2 diabetes mellitus; the case of Jordan
}

\author{
Nahla Khawaja' ${ }^{1}$ Jawad Abu-Shennar ${ }^{1}$, Mohammed Saleh², Said S. Dahbour², Yousef S. Khader ${ }^{3}$ \\ and Kamel M. Ajlouni ${ }^{*}$
}

\section{Correction to: Diabetol Metab Syndr (2018) 10:8 https://doi.org/10.1186/s13098-018-0309-6}

It has been highlighted that the original article [1], published 21 February 2018, contained some errors in Table 3. This correction article shows the correct version of Table 3 and the changes that were made.

\section{Changes:}

1. A labeling error in Table 3 was corrected (Neuropathy status was replaced by Peripheral neuropathy).

2. [Without DPN $n=607(100 \%)$ ] was replaced by (Yes $\mathrm{n}=396)$.

3. [With DPN $n=396$ (100\%)] was replaced by (No $\mathrm{n}=607)$.
4. [Age group (year): (mean $\pm S D)$ ] was replaced by [Age (year)], and the mean age was removed from the table.

5. [Body mass index (BMI) $\left(\mathrm{kg} / \mathrm{m}^{2}\right):(\operatorname{mean} \pm \mathrm{SD})$ ] was replaced by [Body mass index $\left(\mathrm{kg} / \mathrm{m}^{2}\right)$ ] and the mean BMI was removed.

6. [Duration of diabetes (year): (mean $\pm S D)]$ was replaced by [Duration of diabetes (year)] and the mean duration was removed.

7. SBP $\mathrm{mmHg}($ mean \pm SD) DBP $\mathrm{mmHg}($ mean \pm SD) was removed.

8. All these were removed TC (mean $\pm S D)$, LDL (mean $\pm \mathrm{SD})$, HDL (mean $\pm \mathrm{SD})$. 
Table 3 The prevalence of peripheral neuropathy among patients with type 2 diabetes mellitus in Jordan according to relevant socio-demographic, clinical and laboratory characteristics

\begin{tabular}{|c|c|c|c|}
\hline \multirow[t]{2}{*}{ Variables } & \multicolumn{2}{|c|}{ Peripheral neuropathy } & \multirow[t]{2}{*}{$P$ value } \\
\hline & $\begin{array}{l}\text { Yes } \\
n=396\end{array}$ & $\begin{array}{l}\text { No } \\
n=607\end{array}$ & \\
\hline Gender & & & 0.137 \\
\hline Male & $201(41.9)$ & $279(58.1)$ & \\
\hline Female & $195(37.3)$ & $328(62.7)$ & \\
\hline Age (year) & & & $<0.001$ \\
\hline$<50$ & $23(17.4)$ & $109(82.6)$ & \\
\hline $50-69$ & $269(38.9)$ & $423(61.1)$ & \\
\hline$\geq 70$ & $104(58.1)$ & 75 (41.9) & \\
\hline Marital status & & & 0.777 \\
\hline Single/divorced/widowed & $52(40.6)$ & $76(59.4)$ & \\
\hline Married & $344(39.3)$ & $531(60.7)$ & \\
\hline Working status & & & $<0.001$ \\
\hline Unemployed & $175(50.9)$ & $169(49.1)$ & \\
\hline Employed & $83(29.6)$ & $197(70.4)$ & \\
\hline Retired & $138(36.4)$ & $241(63.6)$ & \\
\hline Physical activity & & & $<0.001$ \\
\hline Regular & $29(31.5)$ & $63(68.5)$ & \\
\hline Not regular & $72(25)$ & $216(75)$ & \\
\hline No physical activity & $295(47.4)$ & $328(52.6)$ & \\
\hline Family history of diabetes & & & 0.014 \\
\hline Present & $356(41)$ & $513(59)$ & \\
\hline Absent & $40(29.9)$ & $94(70.1)$ & \\
\hline Regular visit to treating physicians & & & $<0.001$ \\
\hline Yes & $342(37.3)$ & $575(62.7)$ & \\
\hline No & $54(62.8)$ & $32(37.2)$ & \\
\hline Body mass index $\left(\mathrm{kg} / \mathrm{m}^{2}\right)$ & & & 0.052 \\
\hline Normal & $32(37.6)$ & $53(62.4)$ & \\
\hline Over weight & $103(34.1)$ & $199(65.9)$ & \\
\hline Obese & $261(42.4)$ & $355(57.6)$ & \\
\hline Duration of diabetes (year) & & & $<0.001$ \\
\hline$<5$ & $29(8.7)$ & $304(91.3)$ & \\
\hline $5-11$ & $139(39)$ & $217(61)$ & \\
\hline$\geq 12$ & $288(72.6)$ & $86(27.4)$ & \\
\hline Having hypertension & & & $<0.001$ \\
\hline Yes & $374(43.5)$ & $486(56.5)$ & \\
\hline No & $22(15.4)$ & $121(84.6)$ & \\
\hline Nephropathy & & & $<0.001$ \\
\hline Yes & $65(69.1)$ & $29(30.9)$ & \\
\hline No & $331(36.4)$ & $578(63.6)$ & \\
\hline Cardiovascular disease & & & $<0.001$ \\
\hline Yes & $210(54.8)$ & $173(45.2)$ & \\
\hline No & $186(30)$ & $434(70)$ & \\
\hline Dyslipidemia & & & $<0.001$ \\
\hline Yes & $381(43)$ & $505(57)$ & \\
\hline No & 15 (12.8) & $102(87.2)$ & \\
\hline
\end{tabular}

Table 3 continued

\begin{tabular}{|c|c|c|c|}
\hline \multirow[t]{2}{*}{ Variables } & \multicolumn{2}{|c|}{ Peripheral neuropathy } & \multirow[t]{2}{*}{$P$ value } \\
\hline & $\begin{array}{l}\text { Yes } \\
n=396\end{array}$ & $\begin{array}{l}\text { No } \\
n=607\end{array}$ & \\
\hline Retinopathy & & & $<0.001$ \\
\hline Yes & $132(67.3)$ & $64(32.7)$ & \\
\hline No & $264(32.7)$ & $543(67.3)$ & \\
\hline Type of treatment & & & $<0.001$ \\
\hline Insulin only & $77(60.2)$ & $51(39.8)$ & \\
\hline Oral hypoglycemia agents only & $120(22.8)$ & $407(77.2)$ & \\
\hline $\begin{array}{l}\text { Oral hypoglycemia agents and } \\
\text { insulin }\end{array}$ & $199(57.2)$ & $149(42.8)$ & \\
\hline $\mathrm{HbA1c}(\%)$ & & & $<0.001$ \\
\hline Controlled $<7 \%$ & $96(22.7)$ & $326(77.3)$ & \\
\hline Uncontrolled $\geq 7 \%$ & $300(51.6)$ & $281(48.4)$ & \\
\hline
\end{tabular}

9. [HbA1C (\%): (mean $\pm \mathrm{SD})]$ was replaced by $[\mathrm{HbA1c}$ (\%)], and the mean HbA1c was removed from the table.

\section{Author details}

${ }^{1}$ National Center (Institute) for Diabetes, Endocrinology and Genetics (NCDEG), The University of Jordan, P.O. Box 13165, Amman 11942, Jordan. ${ }^{2}$ The University of Jordan, P.O. Box 13165, Amman 11942, Jordan. ${ }^{3}$ Jordan University of Science and Technology, P.O. Box 22110, Irbid, Jordan.

The original article can be found online at https://doi.org/10.1186/ s13098-018-0309-6.

\section{Publisher's Note}

Springer Nature remains neutral with regard to jurisdictional claims in published maps and institutional affiliations.

Received: 11 April 2018 Accepted: 11 April 2018

Published online: 18 May 2018

\section{Reference}

1. Khawaja N, Abu-Shennar J, Saleh M, Dahbour SS, Khader YS, Ajlouni KM. The prevalence and risk factors of peripheral neuropathy among patients with type 2 diabetes mellitus; the case of Jordan. Diabetol Metab Syndr. 2018;10:8. https://doi.org/10.1186/s13098-018-0309-6. 